\title{
On the signature and Euler characteristic of certain four-manifolds
}

\author{
By F. E. A. JOHNSON \\ Department of Mathematics, University College London, Gower Street, \\ London WC1E $6 B T$, United Kingdom
}

AND D. KOTSCHICK $†$

Mathematisches Institut, Universität Basel, Rheinsprung 21, 4051 Basel, Switzerland

(Received 6 January 1993; revised 12 February 1993)

Let $M$ be a smooth closed connected oriented 4-manifold; we shall say that $M$ satisfies Winkelnkemper's inequality when its signature, $\sigma(M)$, and Euler characteristic, $\chi(M)$, are related by $\quad|\sigma(M)| \leqslant \chi(M)$.

This inequality is trivially true for manifolds $M$ with first Betti number $b_{1}(M) \leqslant 1$.

Winkelnkemper's theorem [10], re-proved below, is that (1) is satisfied when the fundamental group $\pi_{1}(M)$ is Abelian. In this note we generalize Winkelnkemper's result to more general fundamental groups. We shall also see that most manifolds with a geometric structure satisfy Winkelnkemper's inequality.

Except where geometric structures enter in Section 1, we could consider topological manifolds instead of smooth ones.

\section{Geometric considerations}

We begin by observing that passage to a finite $n$-sheeted covering has the effect of multiplying both the signature and the Euler characteristic by $n$, so that the following is true:

Proposition 1. Let $M$ be a closed connected oriented 4-manifold, and let $N \rightarrow M$ be a finite connected covering; then $M$ satisfies Winkelnkemper's inequality (1) if and only if $N$ does.

Proposition 2. Let $M$ be a connected sum $M=M_{1} \# M_{2}$ of closed connected oriented 4manifolds $M_{1}, M_{2}$. If both, $M_{1}$ and $M_{2}$ satisfy Winkelnkemper's inequality (1), and if the inequality is strict for at least one of the two, then $M$ also satisfies Winkelnkemper's inequality.

Proof. We assume that the inequality (1) is strict for $M_{2}$. As the signature and the Euler characteristic have the same parity, we have $\left|\sigma\left(M_{2}\right)\right| \leqslant \chi\left(M_{2}\right)-2$. This, together with Winkelnkemper's inequality for $M_{1}$, implies $|\sigma(M)|=\left|\sigma\left(M_{1}\right)+\sigma\left(M_{2}\right)\right| \leqslant$ $\left|\sigma\left(M_{1}\right)\right|+\left|\sigma\left(M_{2}\right)\right| \leqslant \chi\left(M_{1}\right)+\chi\left(M_{2}\right)-2=\chi(M)$.

Remark 1. The assumption that the inequality is strict for one summand is

$\dagger$ D. Kotschick would like to thank the Mathematics Institute at the University of Warwick for its hospitality while this work was being done. 
necessary, because the connected sum of two manifolds with signature and Euler characteristic zero does not satisfy Winkelnkemper's inequality.

Theorem 1 (Hitchin [3]). If $M$ is a smooth closed connected oriented 4-manifold which admits an Einstein metric, then $|\sigma(M)| \leqslant \frac{2}{3} \chi(M)$. A fortiori, $M$ satisfies Winkelnkemper's inequality (1).

Proof. If $g$ is any Riemannian metric on $M$, with scalar curvature $s$, Weyl tensor $W$ and traceless Ricci tensor $R i c_{0}$, then Chern-Weil theory gives the following formulae:

$$
\begin{gathered}
\sigma(M)=\frac{1}{12 \pi^{2}} \int_{M}\left|W_{+}\right|^{2}-\left|W_{-}\right|^{2}, \\
\chi(M)=\frac{1}{8 \pi^{2}} \int_{M} \frac{1}{24} s^{2}+|W|^{2}-\left|R i c_{0}\right|^{2} .
\end{gathered}
$$

If $g$ is Einstein, then $R i c_{0}=0$, so that the desired inequality follows.

Theorem 2. A connected compact complex surface satisfies Winkelnkemper's inequality (1) if and only if it is not ruled over a curve of genus $g \geqslant 2$.

Proof. Using $c_{1}^{2}(M)=3 \sigma(M)+2 \chi(M)$ and $c_{2}(M)=\chi(M)$ together with the RiemannRoch formula $\chi\left(\mathcal{O}_{M}\right)=\frac{1}{12}\left(c_{1}^{2}(M)+c_{2}(M)\right)$, the inequality $-\sigma(M) \leqslant \chi(M)$ is equivalent to $\chi\left(\mathcal{O}_{M}\right) \geqslant 0$, where $\chi\left(\mathcal{O}_{M}\right)$ is the holomorphic Euler characteristic. By the EnriquesKodaira classification, this last inequality holds if and only if $M$ is not ruled over a curve of genus $g \geqslant 2$.

Using the same substitution, the other half of Winkelnkemper's inequality, $\sigma(M) \leqslant$ $\chi(M)$, is equivalent to $c_{1}^{2}(M) \leqslant 5 c_{2}(M)$. For surfaces not ruled over curves of genus $g \geqslant 2$, this follows from the Miyaoka-Yau inequality $c_{1}^{2}(M) \leqslant 3 c_{2}(M)$ and $c_{2}(M) \geqslant 0$.

A geometric structure in the sense of Thurston is a locally homogeneous Riemannian metric. In dimension 4 there are 18 types of such structures which occur on closed manifolds; cf. [8]. All but one occur only on manifolds which satisfy (1):

Theorem 3. A connected closed oriented 4-manifold with a geometric structure in the sense of Thurston satisfies Winkelnkemper's inequality (1) if and only if the geometry is not modelled on $\mathrm{S}^{2} \times \mathrm{H}^{2}$.

Proof. This follows by inspecting the 18 different cases, using the calculations of signatures and Euler characteristics given in [8], theorem 6.1. The correction in [6] is crucial here.

Remark 2. The exceptional cases in Theorems 2 and 3 are the same, namely $S^{2}$ bundles over hyperbolic Riemann surfaces.

Example 1. A class of manifolds covered by each of Theorems 1,2 and 3, is that of compact quotients of the unit disc in $\mathbb{C}^{2}$ by a group $\Gamma$ of automorphisms. These are aspherical manifolds of the form $\Gamma \backslash P S U(2,1) / U(2)$.

\section{Algebraic considerations}

We find it convenient to use the following shorthand:

Definition 1. A group $\Gamma$ is a $W$-group if it admits a finite presentation and if 
Winkelnkemper's inequality (1) holds for all smooth closed connected oriented 4manifolds $M$ with $\pi_{1}(M) \cong \Gamma$.

Example 2. All groups with first Betti numbers $b_{1}(\Gamma) \leqslant 1$ are $W$-groups. On the other hand, the free group on two generators (cf. Remark 4 below) and surface groups (cf. Remark 2) are not $W$-groups.

Recall that two groups $\Gamma_{1}$ and $\Gamma_{2}$ are commensurable when there are subgroups $\Delta_{i} \subset \Gamma_{i}$ of finite index such that $\Delta_{1} \cong \Delta_{2}$. From Proposition 1 and the classification of finite coverings we obtain:

Proposition 3. Let $\Gamma_{1}$ and $\Gamma_{2}$ be commensurable finitely presentable groups; then $\Gamma_{1}$ is a W-group if and only if $\Gamma_{2}$ is.

Theorem 4. Suppose that the finitely presentable group $\Gamma$ satisfies the following condition:

$\Gamma$ contains an infinite sequence of subgroups $\left\{\Gamma_{n}\right\}$ of finite index

$$
\text { for which the first Betti number } b_{1}\left(\Gamma_{n}\right)=\operatorname{dim}_{\mathbf{R}} H_{1}\left(\Gamma_{n}, \mathbb{R}\right) \text { is bounded. }
$$

Then $\Gamma$ is a W-group.

Proof. Let $M$ be a smooth closed connected oriented 4-manifold with $\pi_{1}(M) \cong \Gamma$. We denote by $M_{n}$ the finite connected covering of $M$ with $\pi_{1}\left(M_{n}\right) \cong \Gamma_{n}$. In view of the above Proposition it suffices to verify (1) for any one $M_{n}$.

By hypothesis on the family $\left\{\Gamma_{n}\right\}$, there exists an upper bound on the sequence $\left\{b_{1}\left(M_{n}\right)\right\}$. By passing to an infinite subsequence, if necessary, we may assume that it is actually constant. Now we will prove (1) for $M_{1}$.

Let $\alpha_{n}$ denote the index of $\Gamma_{n}$ in $\Gamma$. Since a finitely generated group has only finitely many subgroups of any given finite index, it follows that $\lim _{n \rightarrow \infty} \alpha_{n}=+\infty$.

We denote by $b_{2}^{+}\left(M_{n}\right)$ (respectively $b_{2}^{-}\left(M_{n}\right)$ ) the maximal dimension of a positive (respectively negative) definite subspace of the cup-product form on $H^{2}\left(M_{n}, \mathbb{R}\right)$. From the multiplicativity of the signature and Euler characteristic under passage to finite coverings we have $\chi\left(M_{n}\right)-\sigma\left(M_{n}\right)=\alpha_{n}\left(\chi\left(M_{1}\right)-\sigma\left(M_{1}\right)\right)$. Using $b_{1}\left(M_{n}\right)=b_{1}\left(M_{1}\right)$ we obtain

$$
\frac{b_{2}^{-}\left(M_{n}\right)}{\alpha_{n}}=\left(1-\frac{1}{\alpha_{n}}\right)\left(1-b_{1}\left(M_{1}\right)\right)+b_{2}^{-}\left(M_{1}\right)
$$

The right-hand side converges to $1-b_{1}\left(M_{1}\right)+b_{2}^{-}\left(M_{1}\right)=\frac{1}{2}\left(\chi\left(M_{1}\right)-\sigma\left(M_{1}\right)\right)$ as $\alpha_{n} \rightarrow \infty$. The left-hand side is non-negative for all $n$, so that $\sigma\left(M_{1}\right) \leqslant \chi\left(M_{1}\right)$ follows.

Similarly, starting from $\chi\left(M_{n}\right)+\sigma\left(M_{n}\right)=\alpha_{n}\left(\chi\left(M_{1}\right)+\sigma\left(M_{1}\right)\right)$, we obtain $-\sigma\left(M_{1}\right) \leqslant$ $\chi\left(M_{1}\right)$.

Remark 3. A similar result appears in [2], page 83. This was pointed out to us by an anonymous referee of the first version of this paper.

The class of groups satisfying condition (4) is closed under extension. More generally, we have:

Proposition 4. Suppose that the finitely presentable group $\Gamma$ occurs in an extension

$$
1 \rightarrow K \rightarrow \Gamma \rightarrow \Delta \rightarrow 1
$$


in which $K$ is finitely generated and $\Delta$ satisfies condition (4). Then $\Gamma$ also satisfies (4).

Proof. Let $\left\{\Delta_{n}\right\}$ be an infinite sequence of subgroups of finite index in $\Delta$ for which the sequence $\left\{\operatorname{dim}_{\mathbb{R}} H^{1}\left(\Delta_{n}, \mathbb{R}\right)\right\}$ is bounded, and let $\Gamma_{n}=\phi^{-1}\left(\Delta_{n}\right)$, where $\phi: \Gamma \rightarrow \Delta$ is the homomorphism in the exact sequence (6). Each $\Gamma_{n}$ is a subgroup of finite index in $\Gamma$. From the spectral sequence for the cohomology of $\Gamma_{n}$, we see that

$$
\begin{aligned}
\operatorname{dim}_{\mathbf{R}} H^{1}\left(\Gamma_{n}, \mathbb{R}\right) & =\operatorname{dim}_{\mathbf{R}} H^{1}\left(\Delta_{n}, \mathbb{R}\right)+\operatorname{dim}_{\mathbf{R}}\left(H^{1}(K, \mathbb{R})\right)^{\Delta_{n}} \\
& \leqslant \operatorname{dim}_{\mathbf{R}} H^{1}\left(\Delta_{n}, \mathbb{R}\right)+\operatorname{dim}_{\mathbf{R}} H^{1}(K, \mathbb{R}) .
\end{aligned}
$$

However, by hypothesis on $K, \operatorname{dim}_{\mathbf{R}} H^{1}(K, \mathbb{R})$ is finite, so that the sequence $\left\{\operatorname{dim}_{\mathbb{R}} H^{\mathbf{1}}\left(\Gamma_{n}, \mathbb{R}\right)\right\}$ is bounded. Thus $\Gamma$ satisfies $(4)$.

It is clear that the infinite cyclic group $\mathbb{Z}$ satisfies (4), since it contains an isomorphic copy of itself of index $n$, for each $n$. Thus we have:

CoRollaRY 1. Let $\Gamma$ be a finitely presentable group which occurs in an extension

$$
1 \rightarrow K \rightarrow \Gamma \rightarrow \mathbb{Z} \rightarrow 1
$$

in which $K$ is finitely generated. Then $\Gamma$ satisfies condition (4).

Remark 4. Some hypothesis on the kernel $K$ in the above is obviously necessary; for example $F_{2}$, the free group of rank 2 , occurs in an extension

$$
1 \rightarrow K \rightarrow F_{2} \rightarrow \mathbb{Z} \rightarrow 1
$$

in which the kernel is a free group of infinite rank. However, it follows easily from the Nielsen-Schreier formula for the rank of a subgroup of a free group that $F_{2}$ does not satisfy the condition (4). Moreover, $F_{2}$ is not a $W$-group either, as the example $M=\left(S^{1} \times S^{3}\right) \#\left(S^{1} \times S^{3}\right)$ shows. Here $\pi_{1}(M) \cong F_{2}$ and $\chi(M)=-2<0=\sigma(M)$.

THEOREM 5. Every poly-(finite or cyclic) group is a W-group.

Proof. If $\Gamma$ is poly-(finite or cyclic), then it contains a subgroup of finite index which is poly- $\mathbb{Z}$. Using induction, Theorem 4 implies that every poly- $\mathbb{Z}$ group is a $W$-group.

Since finitely presentable Abelian groups are polycyclic, we obtain:

CoRollaRY 2 (Winkelnkemper[10]). Let $M^{4}$ be a smooth connected closed oriented 4manifold with Abelian fundamental group. Then $|\sigma(M)| \leqslant \chi(M)$.

We proceed to discuss which lattices in linear Lie groups are $W$-groups. Let $G$ be a connected linear Lie group and $\Gamma \subset G$ a discrete subgroup of finite covolume, i.e. a lattice in the sense of [7].

First observe that $G$ contains a unique maximal normal connected soluble subgroup $\operatorname{rad}(G)$ which is closed in $G$; moreover, $G$ contains a closed connected semisimple subgroup $G_{s}$ with the property that $G=\operatorname{rad}(G) \cdot G_{s}$ and such that $\operatorname{rad}(G) \cap G_{s}$ is a finite central subgroup of $G$. If we put $H=G /\left(\operatorname{rad}(G) \cap G_{s}\right)$, then $H$ is a semidirect product of $\operatorname{rad}(H)$ and $H_{s}$, where $\operatorname{rad}(H)=\operatorname{rad}(G) /\left(\operatorname{rad}(G) \cap G_{s}\right)$ and $H_{s}=G_{s} /\left(\operatorname{rad}(G) \cap G_{s}\right)$.

The fact that $\Gamma$ has finite covolume ensures, by a theorem of Selberg, that $\Gamma$ contains a torsion-free subgroup $\Gamma_{0}$ of finite index. Since $\operatorname{rad}(G) \cap G_{s}$ is a finite 
subgroup of $G$, it is easy to see that $\Gamma_{0}$ embeds in $H$ as a lattice. Thus, up to commensurability of $\Gamma$, we can suppose that the Lie group $G$ which contains $\Gamma$ is a semidirect product of its radical, $\operatorname{rad}(G)$, and its semisimple part, $G_{s}$. When the semisimple group $G_{\delta}$ has no compact simple factors, a theorem of Wang[9] guarantees that $\Gamma \cap \operatorname{rad}(G)$ is a lattice in the soluble Lie group $\operatorname{rad}(G)$, and that $\Gamma /(\Gamma \cap \operatorname{rad}(\Gamma))$ projects to a lattice in the semisimple Lie group $G_{s}$; that is, under the hypothesis that $G_{\delta}$ contains no compact factors, we have an exact sequence

$$
1 \rightarrow \Gamma \cap \operatorname{rad}(G) \rightarrow \Gamma \rightarrow \Gamma /(\Gamma \cap \operatorname{rad}(G)) \rightarrow 1 .
$$

Consider first the extreme case when $\Gamma=\Gamma \cap \operatorname{rad}(G)$. Then $\Gamma$, being a lattice in a connected soluble Lie group, is polycyclic. By Theorem $5, \Gamma$ is a $W$-group.

In the other extreme case when $\operatorname{rad}(G)$ is trivial, $\Gamma$ is a torsion free lattice in a connected semisimple linear Lie group $G$ which has no nontrivial compact normal subgroup. Under this hypothesis $G$ decomposes as a product of simple non-compact Lie groups $G=G_{1} \times \ldots \times G_{n}$. The lattice $\Gamma$ is said to be irreducible in $G$ if $\pi_{I}(\Gamma)$ is nondiscrete for every proper non-empty subset $I$ of $\{1, \ldots, n\}$, where

$$
\pi_{I}: G \rightarrow \prod_{i \in I} G_{i}=G_{I}
$$

is the projection. It is a standard consequence of Borel's Density Theorem (cf. [7]) that if $\Gamma$ is not irreducible, there is a unique partition of $\{1, \ldots, n\}$ into non-empty subsets $I_{1}, \ldots, I_{m}$ such that $\Gamma_{1} \times \ldots \times \Gamma_{m}$ has finite index in $\Gamma$, and $\Gamma_{r}$ is irreducible in $G_{I_{r}}$, where $\Gamma_{r}=\Gamma \cap G_{I_{r}}$. The $\Gamma_{r}$ are the irreducible components of $\Gamma$. Suppose that for each $r$, the factor $G_{I_{r}}$ has $\mathbb{R}$-rank $\geqslant 2$. From a theorem of Kahzdan [4] and Wang [9], we conclude $H_{1}\left(\Gamma_{r}, \mathbb{R}\right)=0$. It now follows easily that $H_{1}(\Gamma, \mathbb{R})=0$. This implies that $\Gamma$ is a $W$-group.

Finally, in the general case of $(10), \Gamma \cap \operatorname{rad}(G)$ is finitely generated and, by the argument just given, $\Gamma /(\Gamma \cap \operatorname{rad}(G))$ satisfies condition (4). It follows from Proposition 4 and Theorem 4 that $\Gamma$ is a $W$-group. We have proved:

TheORem 6. If $\Gamma$ is a lattice in a connected linear Lie group $G$ whose semisimple part $G_{s}$ has no compact factors, and such that all the irreducible factors of $\Gamma /(\Gamma \cap \operatorname{rad}(G))$ are of rank $\geqslant 2$, then $\Gamma$ is a $W$-group.

We will see in the next section that a combination of geometric and cohomological arguments gives other examples of lattices which are $W$-groups.

\section{Cohomological considerations}

We say that a finitely presentable group $\Gamma$ has a certain property, like Poincaré duality, or satisfying Winkelnkemper's inequality (1), if its classifying space $K(\Gamma, 1)$ has the said property.

Recall that if $\pi_{1}(M) \cong \Gamma$, then the classifying map $c: M \rightarrow K(\Gamma, 1)$ of the universal covering of $M$ induces a homomorphism of cohomology rings $c^{*}: H^{*}(\Gamma, \mathbb{R}) \rightarrow$ $H^{*}(M, \mathbb{R})$, which is an isomorphism in degrees $\leqslant 1$ and injective in degree 2 .

THEOREM 7. If $\Gamma$ is a group satisfying 3-dimensional oriented Poincaré duality, then $\Gamma$ is a W-group. 
Proof. Let $M$ be any closed oriented 4-manifold with $\pi_{1}(M) \cong \Gamma$. The ring homomorphism $c^{*}$ maps $H^{2}(\Gamma, \mathbb{R})$ isomorphically onto an isotropic subspace for the cup-product form on $H^{2}(M, \mathbb{R})$. We conclude

$$
\min \left\{b_{2}^{-}(M), b_{2}^{+}(M)\right\} \geqslant b_{2}(\Gamma)=b_{1}(\Gamma)=b_{1}(M) .
$$

This implies that $\quad 1-b_{1}(M)+\min \left\{b_{2}^{-}(M), b_{2}^{+}(M)\right\}>0$,

which is equivalent to the strict Winkelnkemper inequality for $M$.

Corollary 3. If $\Gamma$ is the fundamental group of an orientable closed aspherical 3manifold, then $\Gamma$ is a $W$-group.

Example 3. This shows that the fundamental groups of orientable closed hyperbolic 3 -manifolds, i.e. cocompact lattices in $S O(3,1)$, are $W$-groups.

Theorem 8. If $\Gamma$ is a group satisfying 4-dimensional oriented Poincaré duality and Winkelnkemper's inequality (1), then $\Gamma$ is a $W$-group.

Proof. Let $M$ be a closed oriented 4-manifold with $\pi_{1}(M) \cong \Gamma$. For $x, y \in H^{2}(\Gamma, \mathbb{R})$ we have the following identity for the cup-product forms on $M$ and $K(\Gamma, 1)$ :

$$
\left(c^{*}(x), c^{*}(y)\right)=\operatorname{deg}(c)(x, y) .
$$

By changing the orientation of $M$, if necessary, we may assume that $\operatorname{deg}(c) \geqslant 0$.

If $\operatorname{deg}(c)=0$, then $c^{*}$ maps $H^{2}(\Gamma, \mathbb{R})$ isomorphically onto an isotropic subspace for the cup-product form on $H^{2}(M, \mathbb{R})$. We conclude that $\min \left\{b_{2}^{-}(M), b_{2}^{+}(M)\right\} \geqslant b_{2}(\Gamma) \geqslant$ $2 \min \left\{b_{2}^{-}(\Gamma), b_{2}^{+}(\Gamma)\right\}$, which, together with the Winkelnkemper inequality for $\Gamma$, implies the Winkelnkemper inequality for $M$.

If $\operatorname{deg}(c)>0$, then $c^{*}$ maps every positive (respectively negative) definite subspace of $H^{2}(\Gamma, \mathbb{R})$ isomorphically onto a positive (respectively negative) definite subspace of $H^{2}(M, \mathbb{R})$. Thus if the Winkelnkemper inequality holds for $\Gamma$, then it also holds for $M$.

COROLLARY 4. If $\Gamma$ is the fundamental group of an orientable closed aspherical 4manifold satisfying Winkelnkemper's inequality (1), then $\Gamma$ is a W-group.

Combining this with the theorems proved in Section 1 we obtain many examples of $W$-groups:

Example 4. By Theorem 3 the fundamental group of an orientable closed aspherical manifold with a geometric structure in the sense of Thurston is a $W$-group. These groups are of course lattices in the appropriate Lie groups.

Example 5. By Theorem 2 the fundamental group of an aspherical compact complex surface is a $W$-group. Like the previous example, this class contains the lattices mentioned in Example 1. It also contains many examples of groups which are not lattices, for example the extensions of surface groups by surface groups which arise as the fundamental groups of the Atiyah-Kodaira surfaces [1], [5].

\section{REFERENCES}

[1] M. F. Aтиман. The signature of fibre bundles. In Collected papers in honour of K. Kodaira (Tokyo University Press, 1969).

[2] M. Gromov. Volume and bounded cohomology. Publ. Math. IHES 56 (1982), 5-99. 
[3] N. J. Hitchin. Compact four-dimensional Einstein manifolds. J. Differential Geometry 9 (1974), 435-441.

[4] D. A. KahzDaN. On the connection between the dual space of a group and the structure of its closed subgroups. Funct. Anal. Appl. (1967), 63-65.

[5] K. Kodatra. A certain type of irregular algebraic surface. J. Anal. Math. 19 (1967), 207-215.

[6] D. Kotschick. Remarks on geometric structures on compact complex surfaces. Topology 31 (1992), 317-321

[7] M. S. Raghunathan. Discrete subgroups of Lie groups (Springer-Verlag, 1972).

[8] C. T. C. WALL. Geometric structures on compact complex surfaces. Topology 25 (1986) $119-153$.

[9] S. P. WANG. The dual space of semisimple Lie groups. American J. Math. 91 (1969), 921-937.

[10] H. E. Wink ELNKeMPER. Un teorema sobre variedades de dimensión 4. ActaMexicana Ci. Tecn. $2(1968), 88-89$. 